\title{
Behavioral activation for smoking cessation and mood management following a cardiac event: results of a pilot randomized controlled trial
}

Andrew M. Busch ${ }^{1,2,9^{*}}$ (D), Erin M. Tooley ${ }^{3}$, Shira Dunsiger ${ }^{1,4}$, Elizabeth A. Chattillion ${ }^{2,5}$, John Fani Srour ${ }^{2,6}$, Sherry L. Pagoto ${ }^{7}$, Christopher W. Kahler ${ }^{4}$ and Belinda Borrelli ${ }^{8}$

\begin{abstract}
Background: Smoking cessation following hospitalization for Acute Coronary Syndrome (ACS) significantly reduces subsequent mortality. Depressed mood is a major barrier to cessation post-ACS. Although existing counseling treatments address smoking and depression independently in ACS patients, no integrated treatment addresses both. We developed an integrated treatment combining gold standard cessation counseling with behavioral activation-based mood management; Behavioral Activation Treatment for Cardiac Smokers (BAT-CS). The purpose of this pilot randomized controlled trial was to test feasibility, acceptability, and preliminary efficacy of BAT-CS vs. Standard of Care (SC).

Methods: Participants were recruited during hospitalization for ACS and were randomly assigned to BAT-CS or SC. The nicotine patch was offered in both conditions. Smoking, mood, and stress outcomes were collected at end-of-treatment and 24-week follow-up.

Results: Fifty-nine participants (28 BAT-CS, 31 SC) were recruited over 42 weeks, and assessment completion was above $80 \%$ in both conditions. Treatment acceptability and fidelity were high. At 24 week follow-up adjusted odds ratios favoring BAT-CS were 1.27 (95\% Cl: 0.41-3.93) for 7-day point prevalence abstinence and 1.27 (95\% Cl: 0.42-3.82) for continuous abstinence. Time to first smoking lapse was significantly longer in BAT-CS (62.4 vs. 31.8 days, $p=0.03$ ). At 24-weeks, effect sizes for mood and stress outcomes ranged from $\eta_{\text {partial }}^{2}$ of.07-.11, with significant between treatment effects for positive affect, negative affect, and stress.

Conclusions: The design of this study proved feasible and acceptable. Results provide preliminary evidence that combining behavioral activation with standard smoking cessation counseling could be efficacious for this high risk population. A larger trial with longer follow-up is warranted.
\end{abstract}

Trial registration: NCT01964898. First received by clinicaltrials.gov October 15, 2013.

Keywords: Smoking, Cessation, Acute coronary syndrome, Depression, Mood, Behavioral activation

\footnotetext{
* Correspondence: Andrew.Busch@hcmed.org

${ }^{1}$ The Miriam Hospital, Providence, RI, USA

${ }^{2}$ Warren Alpert Medical School of Brown University, Providence, RI, USA

Full list of author information is available at the end of the article
} 


\section{Background}

Smoking cessation following an Acute Coronary Syndrome (ACS) requires increased attention. ACS patients smoke at a significantly higher rate $(29-37 \%$ [1-3]) than the US population (20\% [4]). Without intensive treatment, most patients return to smoking within a year following ACS [5]. Further, ACS patients are at significantly higher risk for recurrent ACS and death if they continue to smoke, with successful smoking cessation reducing mortality by $36 \%[6]$.

Depressed mood is also a major concern following ACS. Clinical and sub-clinical depression are much more common in ACS patients than the general population [7]. Symptoms of depression, even when mild, independently predict post-ACS morbidity and mortality [8]. The American Heart Association now recognizes depression as a major risk factor following ACS [7]. The two cardinal symptoms of clinical depression are negative mood and anhedonia (i.e., lack of interest/pleasure in activities, lack of positive affect). Recent evidence suggests that anhedonia symptoms may be particularly predictive of poor outcomes following ACS, with multiple findings suggesting anhedonia is more predictive than negative mood variables (e.g., [9]).

There are well established bi-directional relationships between smoking and depression/anhedonia in the general population (e.g., [10]). A recent meta analysis of 20 longitudinal studies concluded that those with depression symptoms at the time of ACS had significantly higher smoking rates post-ACS [11]. Further, smoking status partially mediates the relationship between depression and post-ACS mortality [12].

Although there are counseling treatments that address smoking and depression independently in ACS patients, to our knowledge there is no integrated treatment that addresses both. A single, integrated treatment targeting both depressed mood and smoking could be highly effective in reducing post-ACS mortality. Behavioral activation (BA) may be the ideal mood management intervention for such an integrated treatment.

$\mathrm{BA}$ is a counseling intervention that aims to improve mood by re-engaging patients with healthy sources of positive reinforcement in their environment. Patients and counselors work collaboratively to set individually tailored "activation goals" that are pleasurable and/or consistent with the patient's personal goals and values. Activation goals can include social outings, behaviors that advance vocation/education, exercise, hobbies, and explicit health behavior change targets (e.g., activities consistent with smoking cessation [13]).

BA efficacy has been clearly established for the treatment and prevention of depression in psychiatric [14] and medical populations (e.g., $[15,16])$. BA can be delivered with fidelity by nurse-level practitioners [17], directly targets anhedonia [18], and can be effective in relatively few sessions [19]. Most importantly, recent work suggests that BA can facilitate and maintain health behavior changes among those with depressed mood (e.g., [20-22]), including a randomized trial indicating that adding BA-based mood management to standard smoking cessation treatment may be effective for smokers with elevated depression symptoms [23].

\section{Study aims}

We systematically developed a counseling intervention that integrates standard smoking cessation counseling with BA-based mood management for post-ACS smokers; Behavioral Activation Treatment for Cardiac Smokers (BAT-CS). The present study reports the results of a pilot randomized controlled trial comparing the effects of BAT-CS to a Standard-of-Care control on smoking abstinence, as well as mood and stress. Our primary aims were to determine: a) feasibility/acceptability of the trial protocol and $b$ ) if results warrant a fully powered efficacy trial.

\section{Methods}

\section{Participants}

Participants were hospitalized smokers with ACS (diagnosis of unstable angina, ST and non-ST elevation MI) who were smoking before their hospitalization. Inclusion criteria were: 1) ACS diagnosis documented in the medical record, 2) smoking $\geq 3$ cigarettes per day immediately prior to hospitalization, 3) age 18-75, 4) English fluency, 5) regular telephone access, 6) living within a 1 hour drive of the admitting hospital, and 7) willingness to "strongly consider" an attempt to quit smoking at discharge. We used a cut-off of $\geq 3$ cigarettes per day to include regular, daily smokers who could benefit from the nicotine patch. Exclusion criteria were: 1) evidence of limited mental competency, 2) current psychosis, bipolar disorder, borderline personality disorder, or suicidality (based on chart review and self-report), 3) expectation that the participant would not live through the study period, or 4) regularly attending counseling for depression or smoking cessation and plans to continue this counseling after discharge (which would duplicate BATCS treatment).

\section{Sample size and power}

This study was designed as a pilot to examine treatment feasibility/acceptability and preliminary efficacy (i.e., it was not designed to be fully powered or to establish efficacy). Specifically, with an analyzed sample size of 59 this study was underpowered (power $=47 \%$ ) to detect a medium effect (i.e., $\mathrm{OR}=2.50$ ) on dichotomous smoking outcomes. There was greater, (although still not sufficient) power to estimate intervention effects on secondary 
outcomes: there was $75 \%$ power to detect a medium effect (i.e., partial eta squared $=0.13$ ) on longitudinal continuous outcomes and $70 \%$ power to detect a medium effect (i.e., $\mathrm{HR}=0.20)$ on survival analysis outcomes. Thus, primary and secondary outcome results will be presented as preliminary, and we present effect sizes in addition to significance tests. All efficacy outcomes, especially dichotomous outcomes, should be interpreted with caution.

\section{Procedures}

All procedures were approved by The Miriam Hospital institutional review board. Participants were recruited from inpatient cardiac units at The Miriam and Rhode Island Hospitals in Providence, RI during 2013-14. Potential participants were first identified through electronic medical record reviews and medical staff referrals. Patients appearing to meet study criteria were approached by a research assistant regarding participation during their inpatient stay for ACS. Interested patients were screened for eligibility. Those that passed screening and remained willing to participate provided signed informed consent.

\section{In-hospital smoking cessation counseling}

All participants received one 50-min smoking cessation session in the hospital, which did not include any BA or mood management content. This session was guided by the latest version of the Treating Tobacco Use and Dependence Clinical Guidelines [24] and a National Cancer Institute self-help workbook (Clearing the Air) which the participant kept. During this session, counselors provided a strong and personalized recommendation to quit and discussed: 1) past attempts to quit, reframing attempts as learning opportunities, 2) risks of smoking and benefits of quitting in general and for cardiac patients, 3) personal reasons for quitting, 4) how to recognize and avoid smoking triggers, 5) how to elicit support for quitting, 6) tips for managing cravings, 7) planning for a quit date, and 8) safety/efficacy of the nicotine patch. Per treatment guidelines [24], brief motivational strategies were provided to those who remained ambivalent about quitting.

Eight weeks of the nicotine patch were offered at discharge if the patient was willing to make a quit attempt and the patient's physician approved. While use of the nicotine patch appears to be generally safe for cardiac patients [25], there are reports of increased risk to subgroups of ACS patients [26], and clinical practice guidelines [24] recommend that the patch is "used with caution" in the 2 weeks following myocardial infarction. Thus, physician approval was obtained before patch provision. Dosing followed manufacturer recommendations (e.g., starting on $21 \mathrm{mg}$ patch for those smoking $>10$ per day and on $14 \mathrm{mg}$ for those smoking $\leq 10$ per day). All participants who expressed interested in using the patch were cleared by their physician for patch use.

\section{Randomization}

We used a computer generated (using R, Cran.R-project.org) permuted block randomization procedure, with small, random sized blocks. Randomization was stratified by counselor and elevated symptoms of depression (i.e., Patient Health Questionnaire-9 (PHQ-9) $\geq 10$ vs. PHQ-9 $\leq 9$ ). The study statistician provided sequenced randomization envelopes. The randomization envelopes were opened by counselors following the completion of each in-hospital smoking cessation session. Counselors then immediately informed the participant of their treatment condition.

\section{Standard-of-care (SC)}

The SC group received 5 mailings of print materials at 1 , 3, 6, 9 and 12 weeks post-discharge. Materials were 10 smoking cessation educational brochures produced by JourneyWorks Publishing (two sent at each time point). The 10 brochures are: 1) Relapse Happens 2) Breaking Nicotine Addiction, 3) 50 Things You Should Know About Quitting, 4) How Quitting Smoking Affects Your Body, 5) Quit Smoking Without Gaining Weight, 6) How Quitting Smoking Helps Your Heart, 7) Top Ten Steps to Quitting Smoking, 8) The Health Consequences of Smoking, 9) How to Quit When You Have Tried Before, and 10) Adjusting to a Smoke Free Life. SC participants were contacted by a master's level health educator for brief (5-10 min) "check-in" calls following each mailing. Calls focused on whether the participant received and read the written materials, general encouragement to quit smoking, and participant questions about nicotine patch use. There was no discussion of the importance of depressed mood or goal setting in the SC condition.

The treatment provided in the SC condition is above and beyond that provided by most US hospitals. However, the Joint Commission on Hospital Accreditation recently released a new performance standard [27] which encourages US hospitals to provide cessation counseling before discharge, offer cessation pharmacotherapy at discharge if medically appropriate, and assess smoking status and provide some cessation support in the weeks after discharge. SC meets all aspects of the Joint Commission standard. Our choice of a strong control group likely lowered our between group effect on smoking cessation, however it provides a more clinically and scientifically meaningful comparison.

Behavioral activation treatment for cardiac smokers (BAT-CS) The BAT-CS manual was informed by the manual used in the only published RCT of BA as an aid for smoking cessation, as well as an ongoing RCT (R01DA018730) 
[23]. This existing manual was designed for nonhospitalized smokers and was delivered in several 90 min in-person group sessions in a community clinic. Our adaptation of this existing manual was required to account for treatment setting (e.g., initiation while hospitalized) and structure (i.e., fewer, shorter, individual counseling sessions, mostly over the phone) to better fit the needs and preferences of post-ACS patients. We assessed needs and preferences of post-ACS smokers through extensive mixed method interviews [28] and test cases. Adaptation was also guided by clear meta-analytic data on the dose of treatment needed for significant effects on smoking cessation in hospitalized cardiac patients $[29,30]$.

BAT-CS combines standard, guideline-driven smoking cessation counseling with BA-based mood management and goal setting techniques. BA has its underpinnings in the behavioral model of depression and purports that depression and related problem behaviors, such as smoking, can be reduced by reconnecting patients with healthy sources of positive reinforcement. BA accomplishes this through collaboratively defined between-session "activation goals" (i.e., explicitly scheduled between-session activities). In BAT-CS, activation goals had 3 targets: (1) increasing pleasant and/or meaningful activities in order to improve mood and facilitate smoking cessation, (2) increasing activities consistent with a non-smoking lifestyle (e.g., social outings in non-smoking settings with nonsmoking peers), and (3) developing specific steps to prepare for and initiate a quit attempt. The BAT-CS manual is available by request from the first author [31].

All patients randomized to BAT-CS were offered a minimum of 5 post-discharge contacts at 1, 3, 6, 9, and 12 weeks. Session 2 (1 week post-discharge; $50 \mathrm{~min}$ ) occurred in-person at a research clinic or in the participant's home. Session 2 began with assessment of smoking status and mood (using the 2-item Patient Health Questionnaire, PHQ-2). If the patient was not smoking, the counselor reinforced their efforts, discussed successful strategies used to stay quit, and problem-solved challenges to continued abstinence. If the patient was smoking, any attempt to quit or cut down was reinforced, and relapse causes were discussed. For smoking patients interested in making another quit attempt, the counselor reviewed strategies to deal with triggers and cravings, and a new detailed plan for quitting was collaboratively developed. For smoking patients that were not interested in another quit attempt, brief motivational techniques (as specified in clinical guidelines [24]) were provided.

The counselor then reviewed the importance of addressing depressed mood after a hospitalization for ACS, presented a rationale for $\mathrm{BA}$, and assessed potential targets for activation goals. Content of activation goals was determined through a personal values assessment [32] and discussion of pleasant/meaningful activities restricted due to ACS (post-ACS activity restriction has been linked to poor mood and failure to quit smoking [28]). The counselor and patient collaboratively agreed on 2-4 activation goals to improve/maintain mood and facilitate smoking cessation, to be completed before the next session, and problem-solved barriers to completion. At the end of session, the counselor provided a written list of activation goals and solutions to barriers, and offered to send the participant a between-session email or text message reminder to complete their goals.

Sessions 3-6 (3, 6, 9, and 12 weeks post-discharge; $30 \mathrm{~min}$ ) were conducted by phone to minimize participant burden and followed the same basic format: 1) assess smoking status and depressed mood (using PHQ-2); 2) provide support for continued abstinence or smoking cessation as described in session 2 above; 3) review adherence to activation goals agreed upon in the previous session and assess reasons for failure to complete goals; 4) collaboratively choose new activation goals; and 5) problem-solve barriers to completion of new goals. Patients were offered a between-session email or text message reminder to complete goals. Session 6 (12 weeks post discharge), focused on maintenance of a high rate of behaviors that are pleasant, valued, and consistent with a non-smoking lifestyle following counseling termination.

Up to 4 additional phone booster sessions were offered (i.e. patient could choose to schedule or not) if the participant self-reported high levels of depressed mood (PHQ-2 $\geq 3$ ) or relapse to smoking, or by participant request. The content of booster sessions was similar to follow-up calls but focused on the issue that triggered the booster session (e.g., if prompted by a high PHQ-2, its primary focus was mood management). Booster sessions were scheduled 1 week after any session where high depression or relapse was reported and lasted about $15 \mathrm{~min}$.

\section{Treatment fidelity}

All in hospital sessions and post-randomization BAT-CS counseling sessions were conducted by the first author (licensed clinical psychologist) and second author (clinical psychology post-doctoral fellow supervised by the first author). Note that although PhDs performed all BAT-CS treatment in this pilot trial, manual content was designed for use by bachelor's level counselors.

Attendance was tracked in BAT-CS and engagement with mailed written materials was tracked in SC. Treatment fidelity checklists were completed by counselors following the in-hospital smoking cessation session in both conditions. Following each BAT-CS session, counselors also completed treatment fidelity checklists, recorded activation goals set, and recorded participant self-report of the percent completion of 
each activation goal from the previous session (rated from 0 to $100 \%$ completed).

\section{Assessments \\ Participants completed the baseline assessment while hospitalized. Follow-up assessments were completed at end-of-treatment (12 weeks after hospital discharge) and 24 weeks post-discharge. Assessments were conducted by study staff blind to treatment condition. \\ Sociodemographic, medical status, and smoking history variables}

At baseline (i.e., during hospital stay), participants selfreported socio-demographics and smoking history. Initial medical status and history were obtained through self-report and chart review. Nicotine dependence was measured using the Fagerstrom Test for Nicotine Dependence (FTND [33]).

\section{Feasibility}

Design feasibility was determined by: 1) recruitment rate, 2) percentage of those screened who qualified, 3) percentage of those qualified who chose to participate, and 4) percentage that completed follow-up assessments. Treatment feasibility was determined by number of post-discharge sessions attended in BAT-CS and number of print materials read in SC.

\section{Acceptability}

Treatment acceptability was assessed in both conditions at end-of-treatment using the Client Satisfaction Questionnaire (CSQ [34]), an established measure of patient satisfaction with treatment quality, quantity, and procedures. Scores range from 8 to 32 with higher scores indicating greater satisfaction.

\section{Smoking outcomes}

The primary smoking outcome was 7-day point prevalence abstinence (7-day PPA), defined as no smoking at all in the past 7 days, not even a puff. A breath sample was collected at follow-up assessments if the participant reported $\geq 7$-days of abstinence. Carbon monoxide (CO) level in the breath sample verified self-reported 7-day PPA $(<10 \mathrm{ppm}=$ abstinence $)$. We made an a priori choice of $\mathrm{CO}<10 \mathrm{ppm}$ to verify abstinence as recommended by the most recent Society for Research on Nicotine and Tobacco consensus statement [35]. We are aware of some data suggesting that cutoffs as low as $3 \mathrm{ppm}$ can increase certainty in determining smoking abstinence [36, 37]. However, other work indicates that for patients with Chronic Obstructive Pulmonary Disease (COPD) and other breathing disorders, these lower cutoffs may lead to a high rate of false negatives and that a cutoff of $<10 \mathrm{ppm}$ produces the most accurate results in this population [38]. We knew a priori from our previous pilot data that a significant proportion of our sample in this trial would have COPD, asthma, and/or shortness of breath due to heart failure. Thus, we maintained our a priori cut-off of $\mathrm{CO}<10 \mathrm{ppm}$. Those who reported abstinence from smoking cigarettes but did not provide a breath sample or reported using other tobacco products or electronic cigarettes containing nicotine were considered smokers. We also report continuous abstinence since hospital discharge, and time to first lapse (i.e., first puff) and time to first relapse (i.e., smoking on 7 consecutive days or smoking in 2 consecutive 7 day periods), which were determined through timeline follow back interviewing methods at each assessment.

\section{Mood and stress outcomes}

Depression symptoms were assessed using the Patient Health Questionnaire-9 (PHQ-9) [39]. PHQ-9 scores range from 0 to 27 with higher scores indicating more depression symptoms. The standard cut-off on the PHQ-9 (i.e., $\geq 10$ indicates likely major depression) was used to estimate the rate of major depression at baseline. The ten item Positive Affect Negative Affect Scales (PANAS) [40] assessed positive (PANAS-PA) and negative (PANAS-NA) affect at all time points. PANAS-PA scores range from 5 to 25 with higher scores indicating greater positive affect in the past week. PANAS-NA scores range from 5 to 25 with higher scores indicating greater negative affect in the past week. Perceived stress was measured using the Perceived Stress Scale (PSS [41]); scores range from 0 to 16 with higher scores indicating greater stress over the past month. We included stress as an outcome because it has been linked to poor prognosis [42].

\section{Potential confounders}

We tracked nicotine patch use, engagement in cardiac rehabilitation, and engagement in non-study provided counseling or medication treatment for smoking or depression during the study period. Note that we excluded based on ongoing counseling for smoking cessation or depression at baseline, but for ethical reasons, we did not restrict participants from seeking out additional (i.e., outside the study) counseling treatment during study participation. Thus, we have included non-study counseling for smoking cessation or depression as potential confounders.

\section{BA mechanism}

Behavioral activation (i.e., rate of active, pleasurable, and/or goal directed behavior) was measured using the Behavioral Activation for Depression Scale-Short Form (BADS) [43]. Scores range from 0 to 54 with higher scores indicating greater activation over the past week. 


\section{Data analysis plan}

As a preliminary step in analyzing outcomes, between group differences in baseline characteristics, medical history, and psychosocial variables were compared using $t$ tests, chi-squared tests, and Mann-Whitney U tests. Unadjusted smoking rates and unadjusted mood and stress outcomes were summarized over time by group.

For smoking outcomes we considered nicotine patch use, concurrent smoking counseling, and smoking medication use as potential confounders. For mood and stress outcomes we considered cardiac rehabilitation attendance (because cardiac rehabilitation improves mood), concurrent depression counseling, and concurrent depression medication treatment as potential confounders. Inclusion of these covariates in the final model was determined by model fit.

Using a series of longitudinal regression models implemented with generalized estimating equations (GEEs), we assessed effects of BAT-CS vs. SC on biochemically verified 7-day PPA at end-of-treatment and 24 weeks, as well as the binary indicator of continuous abstinence since hospital discharge at end-of-treatment and 24 weeks, specifying a logit link function. Models included robust standard errors to adjust for repeated measures within participant over time and adjusted for potential confounders identified a priori (final models presented below). Interest was in estimating effects (odds ratios) and corresponding 95\% confidence intervals, rather than strict statistical hypothesis testing.

Secondary smoking outcomes included time to first lapse and time to first relapse. As these outcomes are time to event data, survival analysis was used to model the risk of lapsing/relapsing over time. In this case, each participant contributes two outcome variables to the model: $\mathrm{T}^{*}{ }^{*}$, time to first lapse/relapse and $\mathrm{C}_{\mathrm{i}}$, censoring time. For participants who lapse/relapse before the end of treatment, $\mathrm{T}^{*}{ }_{\mathrm{i}}<\mathrm{C}_{\mathrm{i}}$; for those who don't lapse/relapse before end of treatment or who discontinue the study protocol before lapsing/relapsing, $\mathrm{T}^{*}{ }_{\mathrm{i}}>\mathrm{C}_{\mathrm{i}}$. Thus, the model uses $\mathrm{T}_{\mathrm{i}}=\min \left(\mathrm{T}^{*}{ }_{\mathrm{i}}, \mathrm{C}_{\mathrm{i}}\right)$ as the response for each participant. Using a Cox model, we created the hazard function (which can be thought of as the number of lapses/relapses per patient-day of followup time) as a function of a baseline hazard rate $\lambda_{0}(t)$ and covariates $X(t)$, including treatment assigned and potential confounders.

Next, using a series of longitudinal regression models implemented with GEEs [44] and identity link, we estimated the effects of treatment on mean changes from baseline in PHQ-9, PANAS-PA, PANAS-NA, PSS, and the BADS at end-of-treatment and 24 weeks. Models adjusted for confounders identified a priori and baseline value of the outcome. We also report PHQ-9 models stratified by baseline depression.
Finally, correlations between changes in BADS from baseline to end-of-treatment and 24 weeks and concurrent changes in other mood and stress outcomes were estimated to assess consistency with the BA model. We used spearman rank correlations which are less sensitive to potential outliers, which is important in small samples.

All analyses were carried out using the intent to treat (ITT) sample and run using SAS 9.3. Likelihood based approaches were used for estimation. Models made use of all available data in order to estimate effects, without directly imputing missing values.

\section{Results}

Sample

One hundred thirty-nine patients were approached, 103 were screened for eligibility, 71 were eligible, and 65 consented to participate. One patient's diagnosis was changed to a non-ACS diagnosis before randomization, thus, 64 patients were randomized. Three randomized participants did not receive the intervention (one was unexpectedly discharged to a residential rehabilitation institution with a campus smoking ban for the treatment period, two refused any treatment after discharge), and two were considered ineligible post-randomization because their final discharge diagnosis was not ACS. Thus, we report on the outcomes of 59 participants, 31 who received SC and 28 who received BAT-CS. See Fig. 1 for Consort Diagram which includes reasons for exclusion. Table 1 presents baseline data by treatment condition. No significant differences were observed between groups on any variable in Table 1.

\section{Feasibility and acceptability}

$74.1 \%$ of those approached completed screening. 91.5\% of those who passed screening consented. We recruited for a total of 42 weeks, thereby recruiting 1.4 participants per week that were included in analyses. 93.2 and $84.7 \%$ of participants provided data at end-of-treatment and 24 week assessments respectively (see Fig. 1). BATCS participants completed an average of $4.5(\mathrm{SD}=1.5)$ post-discharge sessions. Booster sessions were utilized by $32.1 \%$ of all BAT-CS participants. SC participants self-reported reading an average of 7.4 $(\mathrm{SD}=2.3)$ of the 10 post-discharge brochures. Mean CSQ satisfaction score was $31.1(\mathrm{SD}=1.3)$ in $\mathrm{SC}$ and $30.5(\mathrm{SD}=2.7)$ in BAT-CS, a non-significant between-group difference.

\section{Treatment Fidelity}

The treatment adherence checklist indicated that counselors provided planned in-hospital smoking cessation treatment components at a rate of $96.9 \%$ in SC and 97.3\% in BAT-CS. There was no significant betweengroup difference in in-hospital session adherence. 


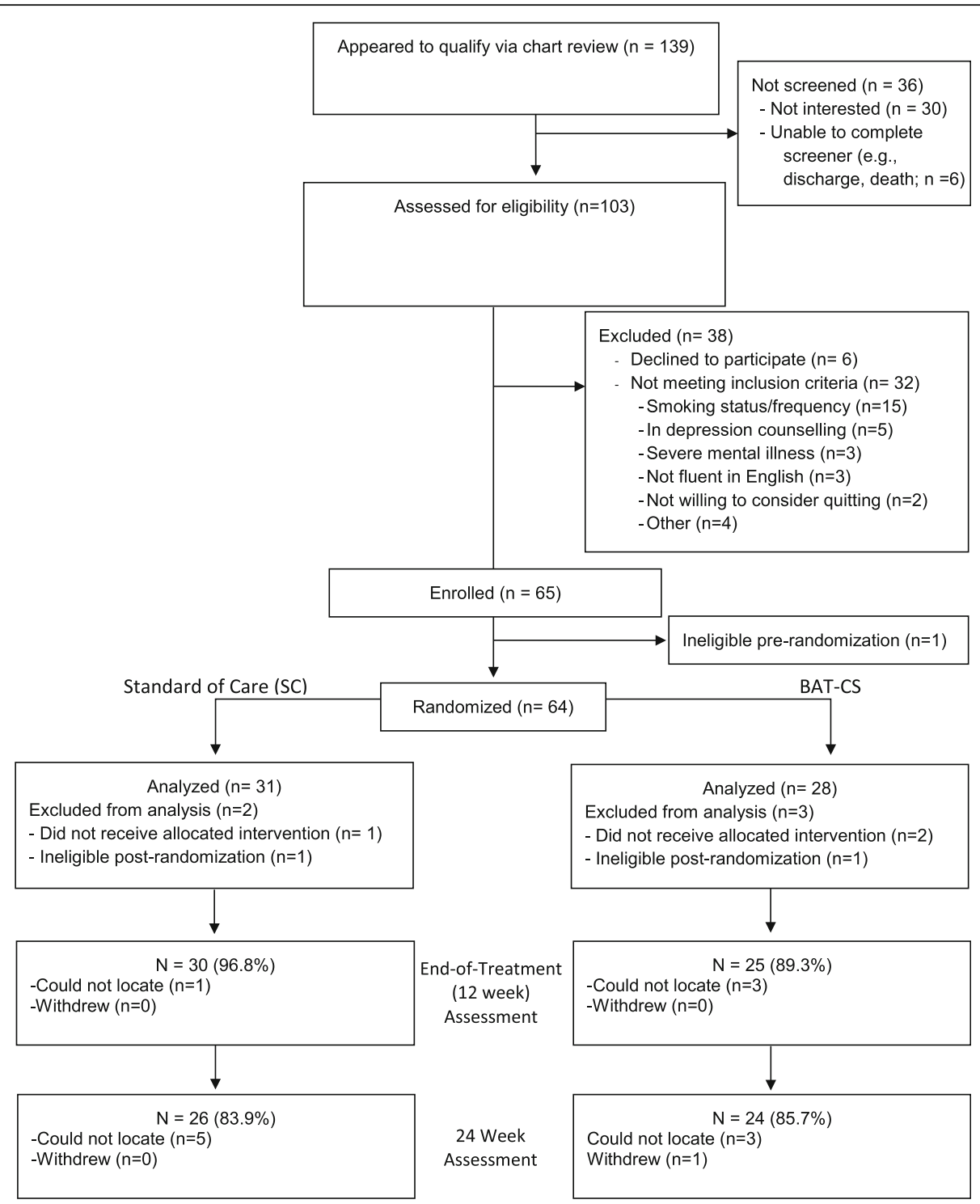

Fig. 1 Consort diagram

Counselors reported providing an average of $97.0 \%$ of planned treatment components during post-discharge BAT-CS sessions. BAT-CS participants set an average 10.6 (SD = 3.6) activation goals during the course of treatment. Average goal completion percentage was $72.7 \%(\mathrm{SD}=14.7 \%)$.

\section{Potential confounders}

There were no significant differences between groups in: 1) use of study-provided nicotine patch (58.1\% SC vs. $67.9 \%$ BAT-CS), 2) use of non-study cessation medication (25.8\% $\mathrm{SC}$ vs. $15.4 \%$ BAT-CS), 3) engagement in concurrent smoking cessation counseling ( $0.0 \%$ for both conditions), 4$)$ attendance at cardiac rehabilitation $(38.7 \%$ SC vs. $38.5 \%$ BAT-CS), 5) use of antidepressants (16.1\% SC vs. $15.4 \%$ BAT-CS), or 6) use of non-study depression counseling
(3.2\% SC vs. 7.7\% BAT-CS). Although there were not between group differences, variables were still considered potential confounders of the treatment effect and were thus included as part of the final model when model fit indicated inclusion.

\section{Smoking outcomes}

Unadjusted smoking outcomes (i.e., without covariates, assessment completers only) indicate that $48.0 \%$ of BATCS vs. $44.8 \%$ of SC participants achieved 7-day PPA at end-of-treatment and that $45.8 \%$ of BAT-CS vs. $42.3 \%$ of SC participants achieved 7-day PPA at 24 week followup. In addition, in unadjusted analyses $44.0 \%$ of BAT-CS vs. $33.3 \%$ of SC participants were continuously abstinent through end-of-treatment and $37.5 \%$ of BAT-CS vs. 
Table 1 Baseline participant characteristics, mean (SD) or \%

\begin{tabular}{|c|c|c|c|}
\hline & BAT-CS $(n=28)$ & $\mathrm{SC}(n=31)$ & Total $(n=59)$ \\
\hline \multicolumn{4}{|l|}{ Demographics } \\
\hline Age (years) & $53.9(11.9)$ & $57.1(8.3)$ & $55.6(10.2)$ \\
\hline Married or living with committed partner & $53.6 \%$ & $58.1 \%$ & $55.9 \%$ \\
\hline Female & $17.9 \%$ & $35.5 \%$ & $27.1 \%$ \\
\hline \multicolumn{4}{|l|}{ Race } \\
\hline Non-Hispanic Caucasian & $89.3 \%$ & $90.3 \%$ & $89.8 \%$ \\
\hline Non-Hispanic African American & $3.6 \%$ & $6.5 \%$ & $5.1 \%$ \\
\hline Hispanic Caucasian & $7.1 \%$ & $0 \%$ & $3.4 \%$ \\
\hline Multiracial & $0 \%$ & $3.2 \%$ & $1.7 \%$ \\
\hline Employed (full or part-time) & $67.8 \%$ & $58.1 \%$ & $62.7 \%$ \\
\hline Median yearly household income ${ }^{a}$ & $\$ 44,000$ & $\$ 35,500$ & $\$ 38,000$ \\
\hline Some college education & $46.4 \%$ & $58.1 \%$ & $47.5 \%$ \\
\hline \multicolumn{4}{|l|}{ Medical History } \\
\hline Prior ACS event & $21.4 \%$ & $35.5 \%$ & $28.8 \%$ \\
\hline \multicolumn{4}{|l|}{ Co-morbidities } \\
\hline Heart Failure & $10.7 \%$ & $16.1 \%$ & $13.6 \%$ \\
\hline Diabetes & $25.0 \%$ & $35.5 \%$ & $30.5 \%$ \\
\hline COPD & $25.0 \%$ & $29.0 \%$ & $27.1 \%$ \\
\hline Previous Stroke & $3.6 \%$ & $3.2 \%$ & $3.4 \%$ \\
\hline Peripheral Artery Disease & $17.8 \%$ & $6.5 \%$ & $11.9 \%$ \\
\hline \multicolumn{4}{|l|}{ ACS Characteristics } \\
\hline \multicolumn{4}{|l|}{ Type of index ACS event } \\
\hline STEMI & $42.9 \%$ & $54.8 \%$ & $49.2 \%$ \\
\hline NSTEMI & $46.4 \%$ & $35.5 \%$ & $40.7 \%$ \\
\hline Unstable Angina & $10.7 \%$ & $9.7 \%$ & $10.2 \%$ \\
\hline \multicolumn{4}{|l|}{ Intervention } \\
\hline Cardiac Catheterization & $100 \%$ & $96.8 \%$ & $98.3 \%$ \\
\hline$\geq 1$ stents placed & $78.6 \%$ & $87.1 \%$ & $83.1 \%$ \\
\hline CABG & $14.3 \%$ & $6.5 \%$ & $10.2 \%$ \\
\hline Length of Hospital Stay (days) & $3.6(4.7)$ & $3.6(3.0)$ & $3.6(3.9)$ \\
\hline LVEF $<55 \%{ }^{a}$ & $46.2 \%$ & $53.3 \%$ & $50.0 \%$ \\
\hline \multicolumn{4}{|l|}{ Baseline Smoking } \\
\hline Cigarettes/day & $15.8(9.2)$ & $16.9(9.5)$ & $16.4(9.3)$ \\
\hline Total years smoking regularly & $35.1(13.9)$ & $41.1(8.8)$ & $38.3(11.8)$ \\
\hline$\geq$ Monthly Other tobacco use & $3.6 \%$ & $9.7 \%$ & $6.8 \%$ \\
\hline$\geq$ Monthly Electronic-Cigarette use & $14.3 \%$ & $9.7 \%$ & $11.9 \%$ \\
\hline FTND & $4.7(2.4)$ & $4.8(2.2)$ & $4.8(2.3)$ \\
\hline \multicolumn{4}{|l|}{ Baseline Mood } \\
\hline Depressive Symptoms (PHQ-9) & $6.8(5.8)$ & $7.0(6.5)$ & $6.9(6.1)$ \\
\hline $\mathrm{PHQ} \geq 10$ & $25.0 \%$ & $29.0 \%$ & $27.1 \%$ \\
\hline Taking Antidepressant Medication & $10.7 \%$ & $9.7 \%$ & $10.2 \%$ \\
\hline
\end{tabular}


Table 1 Baseline participant characteristics, mean (SD) or \% (Continued)

\begin{tabular}{llll}
\hline Positive Affect (PANAS) $^{b}$ & $13.7(4.5)$ & $14.6(4.6)$ & $14.1(19.0)$ \\
Negative Affect (PANAS) $^{b}$ & $9.7(5.1)$ & $7.6(3.6)$ & $8.6(4.5)$ \\
Perceived Stress Scale & $5.5(3.7)$ & $5.4(2.8)$ & $5.5(3.2)$ \\
BADS & $32.7(12.7)$ & $35.9(10.8)$ & $34.4(11.7)$
\end{tabular}

Note. ACS Acute Coronary Syndrome, COPD Chronic Obstructive Pulmonary Disease, STEMI ST segment elevation myocardial infarction, NSTEMI non-ST segment elevation myocardial infarction, CABG Coronary artery bypass graft surgery, LVEF Left ventricular ejection fraction, FTND Fagerstrom Test for Nicotine Dependence, PHQ-9 Patient Health Questionnaire-9, PANAS Positive Affect Negative Affect Scales, BADS Behavioral Activation for Depression Scale-Short Form ${ }^{a} n=56$

$\mathrm{b}_{n}=58$

$34.6 \%$ of SC participants were continuously abstinent through 24 weeks.

Adjusted ITT rates of biochemically verified 7-day PPA and continuous abstinence since hospital discharge at end-of-treatment and 24 week assessments are presented in Fig. 2. There were no significant treatment effects in adjusted outcomes over time. Final models adjusted for nicotine patch use and concurrent medication treatment targeting cessation. At end-of-treatment adjusted odds ratios favoring BAT-CS were $1.12(0.37-3.40)$ for 7 -day PPA and 1.82 (95\% CI: 0.62-5.40) for continuous abstinence. At 24 weeks adjusted odds ratios favoring BAT-CS were 1.27 (95\% CI: 0.41-3.93) for 7-day PPA and 1.27 (95\% CI: 0.42-3.82) for continuous abstinence.

Survival analysis results indicate that mean number of days to first lapse after discharge was significantly greater for BAT-CS vs. SC (62.4 vs. 31.8, $p=0.03)$. When modeling treatment effects on the risk of lapse after discharge,
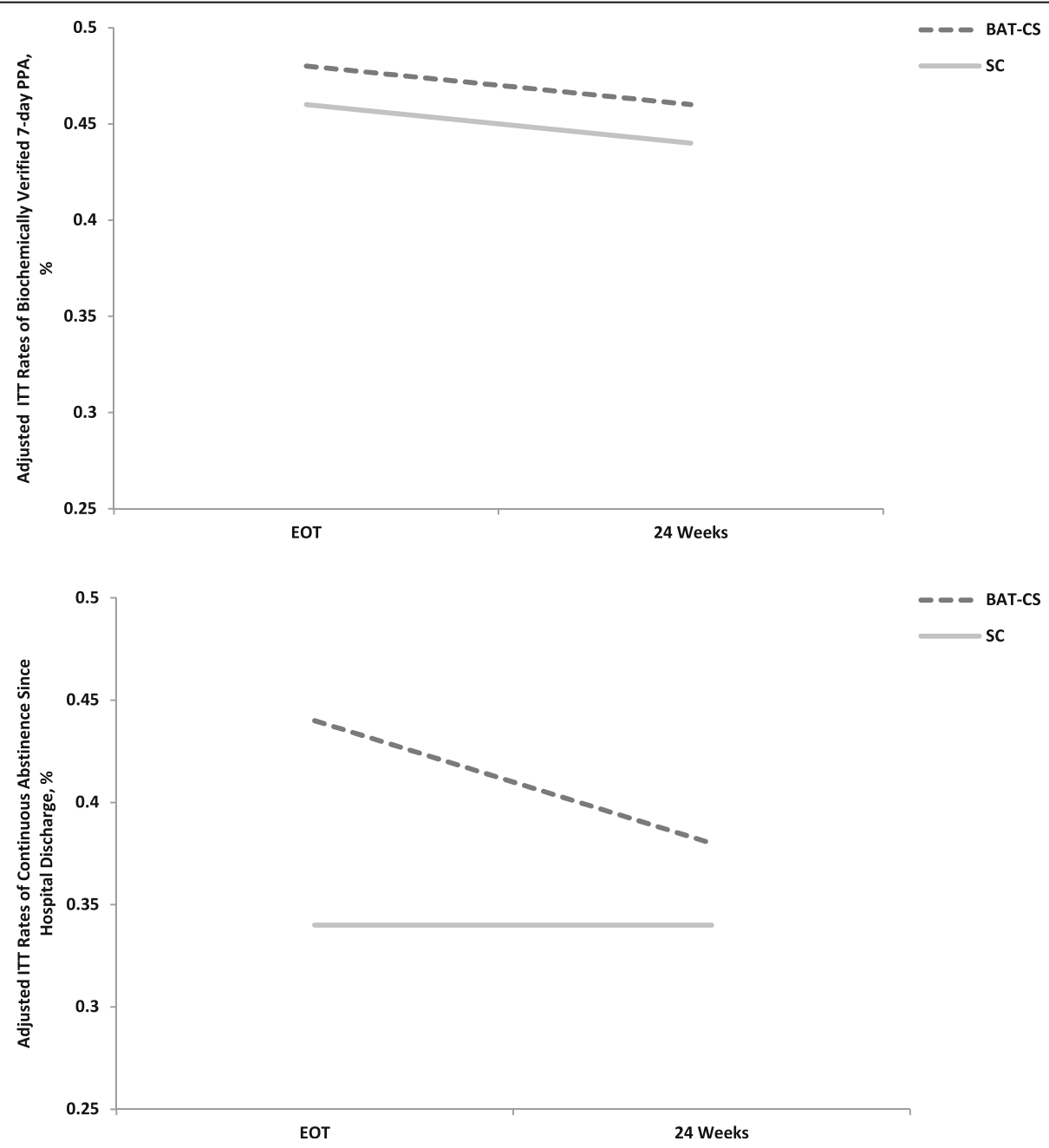

Fig. 2 Adjusted smoking rates over time 
results indicate significant effects, such that for those randomized to BAT-CS, risk of lapse was 0.38 times that of those in SC (HR $=0.38,95 \% \mathrm{CI}: 0.17-0.82, p=0.01)$. Although mean days to first relapse favored BAT-CS participants (71.2 vs. 47.4 days; $p=0.08$ ), there was no significant between-group difference in the risk of relapse $(\mathrm{HR}=0.50,95 \%$ CI: 0.23-1.13, $p=0.10)$.

\section{Mood and stress outcomes}

Additional file 1 presents unadjusted means for all mood and stress outcomes at baseline, end-of-treatment, and 24 weeks. Fig. 3 presents adjusted means for all mood and stress outcomes at baseline, end-of-treatment, and 24 weeks. Exact adjusted means and standard errors for each time point are provided in Additional file 2. Final models for mood and stress outcomes adjusted for antidepressant medication use and for cardiac rehabilitation attendance.

No significant differences between groups were observed for mean change from baseline to end-oftreatment for PANAS-PA $\left(\eta_{\text {partial }}^{2}=0.06, p=0.08\right)$, PANAS-NA $\left(\eta_{\text {partial }}^{2}=0.04, p=0.16\right)$, PSS $\left(\eta_{\text {partial }}^{2}=0.03\right.$, $p=0.35)$, and PHQ-9 $\left(\eta_{\text {partial }}^{2}=0.05, p=0.72\right)$. Effect sizes were modest $\left(\eta_{\text {partial }}^{2} 0.03-0.06\right)$ but in the hypothesized direction favoring BAT-CS. There were significant, small to medium effects favoring BAT-CS on baseline to 24-week changes in PANAS-PA $(\mathrm{b}=2.73, \mathrm{SE}=1.33$, $\left.\eta_{\text {partial }}^{2}=0.10, p=.04\right)$, PANAS-NA $(\mathrm{b}=-3.02, \mathrm{SE}=1.34$, $\left.\eta_{\text {partial }}^{2}=0.11, p=0.02\right)$, and PSS $(\mathrm{b}=-1.80, \mathrm{SE}=0.82$, $\eta_{\text {partial }}^{2}=0.11, p=0.03$ ). No significant effect was observed for change in PHQ-9 from baseline to 24 weeks $\left(\eta_{\text {partial }}^{2}=0.07, p=0.13\right)$, but the effect was in the hypothesized direction favoring BAT-CS.
When comparing effects of treatment on changes in PHQ-9 from baseline to 24 weeks amongst those with and without likely major depression at baseline, results indicate a small, non-significant effect favoring BAT-CS in the PHQ- $9<10$ sub-sample $\left(n=43 ; \eta_{\text {partial }}^{2}=0.09\right.$, $p=0.06)$ and no effect in the PHQ-9 $\geq 10$ sub-sample $\left(n=16 ; \eta_{\text {partial }}^{2}=0.01, p=0.27\right)$.

\section{BA mechanism}

Results indicate a small non-significant between-group effect favoring BAT-CS on change in BADS at end-oftreatment $\left(\eta_{\text {partial }}^{2}=0.05 ; p=0.09\right)$ and 24 weeks $\left(\eta_{\text {par- }}^{2}\right.$ tial $=0.05 ; p=0.11)$. Change in BADS from baseline to end-of-treatment was significantly correlated with concurrent changes in PANAS-PA $\left(\mathrm{r}_{\mathrm{s}}=.27 ; p=.04\right)$, PANAS-NA $\left(\mathrm{r}_{\mathrm{s}}=-.36 ; p<.01\right)$, PHQ-9 $\left(\mathrm{r}_{\mathrm{s}}=-.56\right.$; $p<.001)$, and PSS $\left(\mathrm{r}_{\mathrm{s}}=-.51 ; p<.001\right)$. Change in BADS from baseline to 24-weeks was significantly correlated with concurrent changes in PHQ-9 $\left(\mathrm{r}_{\mathrm{s}}=-.55 ; p<.001\right)$ and PSS $\left(\mathrm{r}_{\mathrm{s}}=-.40 ; p<.01\right)$. Correlations of change in BADS from baseline to 24-weeks with concurrent change in PANAS-PA $\left(\mathrm{r}_{\mathrm{s}}=.23 ; p=.11\right)$ and PANAS-NA $\left(\mathrm{r}_{\mathrm{s}}=-.18 ; p=.22\right)$ were in the hypothesized direction, but were non-significant.

\section{Discussion}

Results support the feasibility of this RCT protocol. Most patients were willing to be screened and most who passed the screener were willing to enroll in the study. We enrolled 1.4 participants per week of recruitment. Over $80 \%$ of participants provided primary outcome data at all assessment time points. CSQ scores indicated good

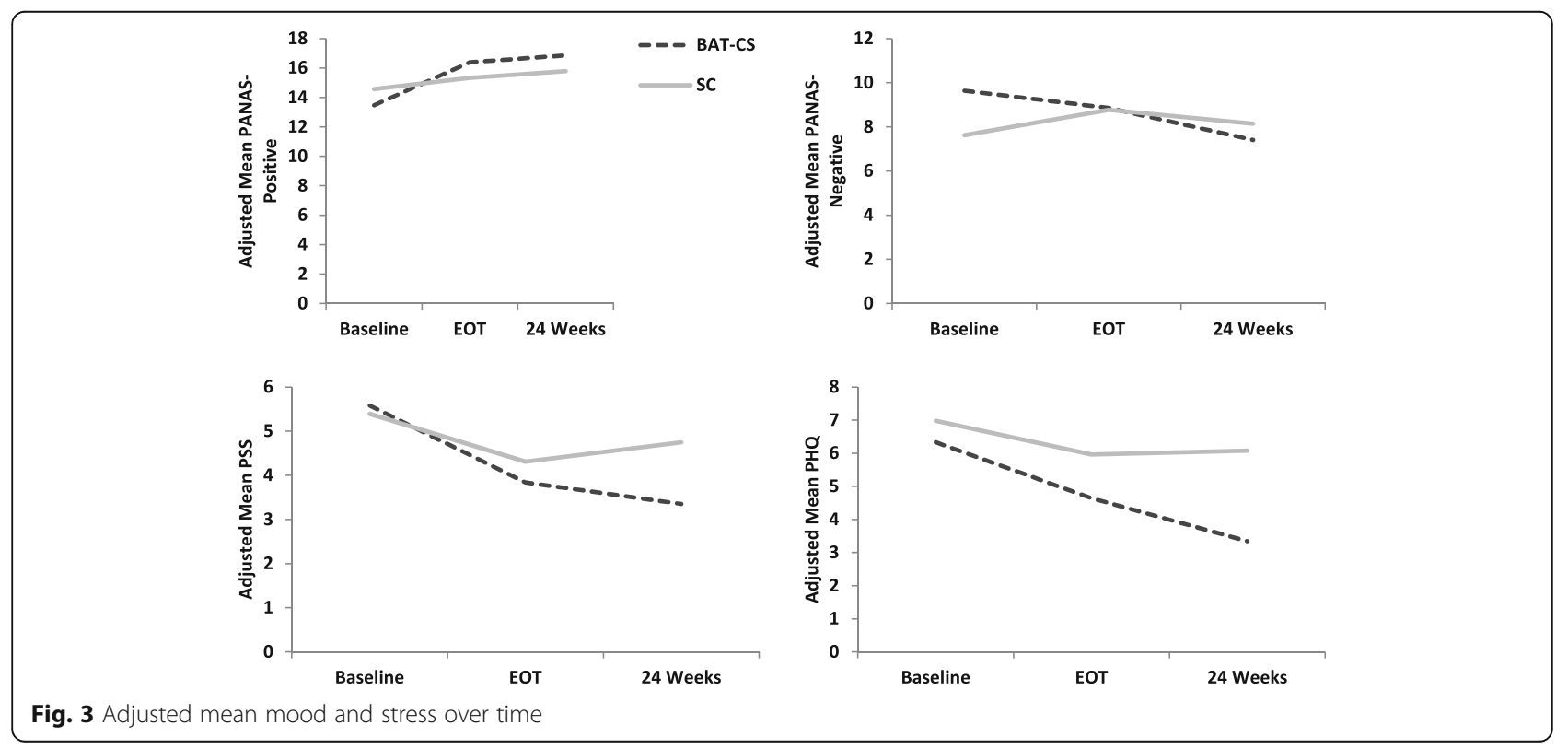


treatment acceptability in both conditions with mean CSQ scores above 30 in both conditions.

All smoking outcomes were in the hypothesized direction, favoring BAT-CS efficacy. However, most were non-significant, which was expected due to limited power in this pilot study. Specifically, in fully controlled models, BAT-CS participants were 1.27 times more likely than SC participants to be both 7-day PPA abstinent and continually abstinent at 24 weeks postdischarge. There were larger effects favoring BAT-CS for continuous abstinence at end-of-treatment $(\mathrm{OR}=1.82)$ and risk of lapse $(\mathrm{HR}=.38, p=.03)$ and risk of relapse $(\mathrm{HR}=.50, p=.10)$ in survival analyses. When evaluating the potential clinical significance of these findings it is important to note that smoking cessation has a direct, proximal effect on mortality in this population [6]. Experts estimate that a cessation treatment that produces a $5 \%$ difference in quit rates in this population would have significant public health value [45]. In adjusted analyses, the current study indicates a 10.3 and $4.3 \%$ between treatment differences in continuous abstinence at endof-treatment and 24-weeks respectively, suggesting potential public health significance. Further, we observed these differences despite providing a robust control condition (including 1 hour of cessation counseling, free nicotine patches, and educational materials).

As hypothesized, depressed mood and stress outcomes favored BAT-CS, with 24-week effect sizes ranging from $\eta_{\text {partial }}^{2}$ of .07 to.11. Differences were statistically significant for positive affect, negative affect, and stress. Change in depression symptoms at 24 weeks was not significantly different between groups, but did show a small to medium effect size favoring BAT-CS. Taken together, results indicate that BAT-CS has promise for improvement in mood and reduction in stress post-ACS. The observed effect on positive affect is particularly promising, as lower positive affect has been prospectively linked to both smoking cessation and post-ACS mortality $[9,10]$.

We chose to enroll patients with a range of baseline depression symptoms (i.e., from those asymptomatic to those with major depression), with the rationale that the immediate post-ACS period is a high risk time for development of depression symptoms [46], especially among smokers [47], and thus many of those without depression at baseline could benefit from BA-based prophylactic mood management. When we examined PHQ-9 changes separately for those who had likely major depression at baseline (PHQ $\geq 10$ at baseline) and those that did not $(\mathrm{PHQ}<10)$, the strongest effect was among those without likely major depression at baseline $\left(\eta_{\text {par- }}^{2}\right.$ tial $=0.09$ ), indicating that BAT-CS may be particularly efficacious for post-ACS depression prevention. This finding is consistent with existing trials showing that
BA-based mood management can prevent depression in medical patients [16] and significantly improve mood in non-depressed individuals [18].

There was almost no 24-week between-group effect on PHQ-9 change among those with PHQ-9 $\geq 10$ at baseline. This needs to be interpreted with caution given the small sample for this analysis $(n=16)$ and existing data indicating that BA-based treatment is effective for major depression in medical patients [15]. It may be the case that those with major depression would benefit from more intensive counseling than was provided in the current study.

BAT-CS had a promising effect $\left(\eta_{\text {partial }}^{2}=0.05\right)$ on BA's purported mechanisms of change measured by the BADS. While not statistically significant, this finding indicates that BA procedures provided in this study are likely affecting the targeted behavior. This result was not surprising given the high goal completion rate observed (73\% in the current study; a previous successful BA for depression trial found a $58 \%$ completion rate using the same homework compliance measure [13]). We also observed correlations in the expected direction between change in BADS and change in mood and stress outcomes consistent with the core of BA theory.

This study has several limitations. First, as a small pilot study, outcome results should be interpreted as preliminary. Second, external validity was limited by the fact that counseling was conducted by Ph.D. providers (which is not the community standard of care) and by a sample that was almost 90\% non-Hispanic Caucasian (which is not representative of the overall racial and ethnic diversity of the U.S.). Third, while our SC control condition exceeds what is generally available in the community, there was still significantly more contact time in BAT-CS. Finally, our longest follow-up was 24 weeks post-ACS, which limited our ability to examine the sustainability of effects.

Despite these limitations, current results are potentially impactful as they are the first data on a combined smoking cessation and mood management counseling intervention post-ACS. These results also add to the growing literature indicating that BA is an effective treatment for depressed mood in medical patients, and this is the second RCT to indicate that BA has promise for the facilitation of smoking cessation. Follow-up trials of BAT-CS should be powered to detect between group differences in mood, verified abstinence, and cardiac health at long-term follow-up and should utilize counselors similar to community providers to increase external validity. Further, future studies could explore the utility of pairing BA with counseling targeting other health behaviors known to be correlated with depression (e.g., sedentary behavior, medication adherence).

\section{Conclusions}

This is the first study to combine smoking cessation and mood management counseling following a cardiac event. 
Results provide preliminary evidence that combining behavioral activation with standard smoking cessation counseling could be efficacious for this high risk population. A larger trial with longer follow-up is warranted.

\section{Additional files} Additional file 1: Unadjusted Mean Mood and Stress Outcomes Over
Time. (DOC $30 \mathrm{~kb}$ )

Additional file 2: Adjusted Mean Mood and Stress Outcomes Over Time.(DOCX $14 \mathrm{~kb})$

\section{Abbreviations}

7-day PPA: 7-day point prevalence abstinence; ACS: Acute Coronary Syndrome; BA: Behavioral activation; BADS: Behavioral Activation for Depression Scale-Short Form; BAT-CS: Behavioral Activation Treatment for Cardiac Smokers; CABG: Coronary artery bypass graft surgery; CO: Carbon monoxide; COPD: Chronic Obstructive Pulmonary Disease; FTND: Fagerstrom Test for Nicotine Dependence; ITT: Intent to treat; LVEF: Left ventricular ejection fraction; NSTEMI: Non-ST segment elevation myocardial infarction; PANAS: Positive Affect Negative Affect Scales; PHQ-9: 9-item patient health questionnaire; SC: Standard of care control group; STEMI: ST segment elevation myocardial infarction

\section{Acknowledgements}

Not applicable.

\section{Funding}

Data collection for this study was supported the National Heart, Lung, and Blood Institute of the National Institutes of Health under award number K23HL107391. The content is solely the responsibility of the authors and does not necessarily represent the official views of the National Institutes of Health.

\section{Availability of data and materials}

Participants only consented to the publication of data in aggregate form. Further, the participants in this study were drawn from a small population (i.e., smokers admitted for ACS at two hospitals across less than a year) which limits our confidence that publishing the raw data reported in this manuscript could be accomplished without compromising the confidentiality of participants. Thus, we are unable to publish this dataset. More detailed summary data is available from the first author to qualified investigators for appropriate purposes (e.g., conducting a meta-analysis). We also offered share more detailed summary data or additional analyses with reviewers of this manuscript if needed for purposes of a thorough review. The trial is registered on the U.S. National Institutes of Health Clinical Trials Registry, NCT01964898.

\section{Authors' contributions}

$A M B, S L P, C W K$, and BB designed the treatment manual and trial protocol. $A M B$ and ET provided treatment. SD conducted statistical analysis. EAC and ET wrote significant portions of the manuscript. EAC conducted study assessments. JFS provided medical consultation on participants, measures, and write up. All authors read and approved the final manuscript.

\section{Competing interests}

The authors declare that they have no competing interests.

\section{Consent for publication}

Not applicable.

\section{Ethics approval and consent to participate}

All procedures were approved by The Miriam Hospital institutional review board (Protocol \#2011-11). All participants signed a written informed consent form.

\section{Publisher's Note}

Springer Nature remains neutral with regard to jurisdictional claims in published maps and institutional affiliations.

\section{Author details}

${ }^{1}$ The Miriam Hospital, Providence, RI, USA. ${ }^{2}$ Warren Alpert Medical School of Brown University, Providence, RI, USA. ${ }^{3}$ Roger Williams University, Bristol, Rl, USA. ${ }^{4}$ Brown University School of Public Health, Providence, RI, USA.

${ }^{5}$ Providence VA Medical Center, Providence, RI, USA. ${ }^{6}$ Rhode Island Hospital, Providence, RI, USA. 'University of Massachusetts Medical School, Worcester, MA, USA. ${ }^{8}$ Boston University, Henry M. Goldman School of Dental Medicine, Boston, MA, USA. ${ }^{9}$ Current correspondence address, Minneapolis Medical Research Foundation, 701 Park Avenue, S9-309, Minneapolis, MN 55415-1623, USA.

Received: 12 July 2016 Accepted: 8 April 2017

Published online: 17 April 2017

\section{References}

1. Khot UN, Khot MB, Bajzer CT, Sapp SK, Ohman EM, Brener SJ, Ellis SG, Lincoff AM, Topol EJ. Prevalence of conventional risk factors in patients with coronary heart disease. JAMA. 2003;290(7):898-904.

2. Centers for Disease Control. Cigarette smoking among adults-United States, 2006. Morb Mortal Wkly Rep. 2007:56:1157-61.

3. Shahoumian TA, Phillips BR, Backus LI. Cigarette smoking, reduction and quit attempts: prevalence among veterans with coronary heart disease. Prev Chronic Dis. 2016;13:E41.

4. Agaku IT, King BA, Dube SR. Centers for disease C, prevention: current cigarette smoking among adults - United States, 2005-2012. Morb Mortal Wkly Rep. 2014;63(2):29-34.

5. Rigotti NA, Munafo MR, Stead LF. Smoking cessation interventions for hospitalized smokers: a systematic review. Arch Intern Med. 2008;168(18):1950-60.

6. Critchley JA, Capewell S. Mortality risk reduction associated with smoking cessation in patients with coronary heart disease: a systematic review. JAMA. 2003;290(1):86-97.

7. Lichtman JH, Froelicher ES, Blumenthal JA, Carney RM, Doering LV, FrasureSmith N, Freedland KE, Jaffe AS, Leifheit-Limson EC, Sheps DS, et al. Depression as a risk factor for poor prognosis among patients with acute coronary syndrome: systematic review and recommendations: a scientific statement from the American Heart Association. Circulation. 2014;129(12): 1350-69.

8. Meijer A, Conradi HJ, Bos EH, Thombs BD, van Melle JP, de Jonge P. Prognostic association of depression following myocardial infarction with mortality and cardiovascular events: a meta-analysis of 25 years of research. Gen Hosp Psychiatry. 2011;33(3):203-16.

9. Davidson KW, Burg MM, Kronish IM, Shimbo D, Dettenborn L, Mehran R Vorchheimer D, Clemow L, Schwartz JE, Lesperance F, et al. Association of anhedonia with recurrent major adverse cardiac events and mortality 1 year after acute coronary syndrome. Arch Gen Psychiatry. 2010;67(5):480-8.

10. Leventhal AM, Piper ME, Japuntich SJ, Baker TB, Cook JW. Anhedonia, depressed mood, and smoking cessation outcome. J Consult Clin Psychol. 2014;82(1):122-9.

11. Doyle F, Rohde D, Rutkowska A, Morgan K, Cousins G, McGee H. Systematic review and meta-analysis of the impact of depression on subsequent smoking cessation in patients with coronary heart disease: 1990 to 2013. Psychosom Med. 2014;76(1):44-57.

12. Brummett BH, Babyak MA, Siegler IC, Mark DB, Williams RB, Barefoot JC. Effect of smoking and sedentary behavior on the association between depressive symptoms and mortality from coronary heart disease. Am J Cardiol. 2003;92(5):529-32.

13. Busch AM, Uebelacker LA, Kalibatseva Z, Miller IW. Measuring homework completion in behavioral activation. Behav Modif. 2010;34(4):310-29.

14. Ekers D, Webster L, Van Straten A, Cuijpers P, Richards D, Gilbody S. Behavioural activation for depression; an update of meta-analysis of effectiveness and sub group analysis. PLoS One. 2014;9(6):e100100.

15. Mitchell PH, Veith RC, Becker K, Buzaitis A, Cain KC, Fruin M, Tirschwell D, Teri L. Brief psychosocial-behavioral intervention with antidepressant reduces poststroke depression significantly more than usual care with antidepressant: living well with stroke: randomized, controlled trial. Stroke. 2009;40(9):3073-8 
16. Rovner BW, Casten RJ, Hegel MT, Massof RW, Leiby BE, Ho AC, Tasman WS Low vision depression prevention trial in age-related macular degeneration: a randomized clinical trial. Ophthalmology. 2014;121(11):2204-11.

17. Ekers DM, Dawson MS, Bailey E. Dissemination of behavioural activation for depression to mental health nurses: training evaluation and benchmarked clinical outcomes. J Psychiatr Ment Health Nurs. 2013;20(2):186-92.

18. Mazzucchelli TG, Kane RT, Rees CS. Behavioral activation interventions for well-being: a meta-analysis. J Posit Psychol. 2010;5(2):105-21.

19. Moore RC, Chattillion EA, Ceglowski J, Ho J, von Kanel R, Mills PJ, Ziegler MG, Patterson TL, Grant I, Mausbach BT. A randomized clinical trial of behavioral activation (BA) therapy for improving psychological and physical health in dementia caregivers: results of the pleasant events program (PEP). Behav Res Ther. 2013;51(10):623-32.

20. Mimiaga MJ, Reisner SL, Pantalone DW, O'Cleirigh C, Mayer KH, Safren SA. A pilot trial of integrated behavioral activation and sexual risk reduction counseling for HIV-uninfected men who have sex with men abusing crystal methamphetamine. AIDS Patient Care STDs. 2012;26(11):681-93.

21. Daughters SB, Braun AR, Sargeant MN, Reynolds EK, Hopko DR, Blanco C, Lejuez CW. Effectiveness of a brief behavioral treatment for inner-city illicit drug users with elevated depressive symptoms: the life enhancement treatment for substance use (LETS act!). J Clin Psychiatry. 2008:69(1):122-9.

22. Daughters SB, Magidson JF, Schuster RM, Safren SA. ACT HEALTHY: a combined cognitive-behavioral depression and medication adherence treatment for HIVinfected substance users. Cogn Behav Pract. 2010;17(3):309-21.

23. MacPherson L, Tull MT, Matusiewicz AK, Rodman S, Strong DR, Kahler CW Hopko DR, Zvolensky MJ, Brown RA, Lejuez CW. Randomized controlled trial of behavioral activation smoking cessation treatment for smokers with elevated depressive symptoms. J Consult Clin Psychol. 2010;78(1):55-61.

24. Fiore MC. Treating tobacco use and dependence: 2008 update. Clinical practice Guidline. Rockville: USDHHS, PHS; 2008.

25. Joseph AM, Norman SM, Ferry LH, Prochazka AV, Westman EC, Steele BG, Sherman SE, Cleveland M, Antonuccio DO, Hartman N, et al. The safety of transdermal nicotine as an aid to smoking cessation in patients with cardiac disease. N Engl J Med. 1996:335(24):1792-8.

26. Paciullo CA, Short MR, Steinke DT, Jennings HR. Impact of nicotine replacement therapy on postoperative mortality following coronary artery bypass graft surgery. Ann Pharmacother. 2009;43(7):1197-202.

27. Fiore MC, Goplerud E, Schroeder SA. The Joint Commission's new tobaccocessation measures-will hospitals do the right thing? N Engl J Med. 2012; 366(13):1172-4.

28. Busch AM, Fani Srour J, Arrighi JA, Kahler CW, Borrelli B. Valued life activities, smoking cessation, and mood in post-acute coronary syndrome patients. Int J Behav Med. 2015;22(5):563-8.

29. Mohiuddin SM, Mooss AN, Hunter CB, Grollmes TL, Cloutier DA, Hilleman DE. Intensive smoking cessation intervention reduces mortality in high-risk smokers with cardiovascular disease. Chest. 2007;131(2):446-52.

30. Rigotti NA, Clair C, Munafo MR, Stead LF. Interventions for smoking cessation in hospitalised patients. Cochrane Database Syst Rev. 2012;5: CD001837.

31. Busch A, Borrelli B, Tooley E: Behavioral activation treatment for cardiac smokers. Unpublished manual. 2013.

32. Lejuez CW, Hopko DR, Acierno R, Daughters SB, Pagoto SL. Ten year revision of the brief behavioral activation treatment for depression: revised treatment manual. Behav Modif. 2011;35(2):111-61.

33. Heatherton TF, Kozlowski LT, Frecker RC, Fagerstrom KO. The Fagerstrom test for nicotine dependence: a revision of the Fagerstrom tolerance questionnaire. Br J Addict. 1991:86(9):1119-27.

34. Nguyen TD, Attkisson CC, Stegner BL. Assessment of patient satisfaction: development and refinement of a service evaluation questionnaire. Eval Program Plann. 1983;6(3-4):299-313.

35. Hughes JR, Keely JP, Niaura RS, Ossip-Klein DJ, Richmond RL, Swan GE. Measures of abstinence in clinical trials: issues and recommendations. Nicotine Tob Res. 2003;5(1):13-25

36. Cropsey KL, Trent LR, Clark CB, Stevens EN, Lahti AC, Hendricks PS. How low should you go? Determining the optimal cutoff for exhaled carbon monoxide to confirm smoking abstinence when using cotinine as reference. Nicotine Tob Res. 2014;16(10):1348-55.

37. Javors MA, Hatch JP, Lamb RJ. Cut-off levels for breath carbon monoxide as a marker for cigarette smoking. Addiction. 2005;100(2):159-67.
38. Sato S, Nishimura K, Koyama H, Tsukino M, Oga T, Hajiro T, Mishima M. Optimal cutoff level of breath carbon monoxide for assessing smoking status in patients with asthma and COPD. Chest. 2003;124(5):1749-54.

39. Kroenke K, Spitzer RL, Williams JB. The PHQ-9: validity of a brief depression severity measure. J Gen Intern Med. 2001;16(9):606-13.

40. Mackinnon A, Jorm AF, Christensen H, Korten AE, Jacomb PA, Rodgers B. A short form of the positive and negative affect schedule: evaluation of factorial validity and invariance across demographic variables in a community sample. Personal Individ Differ. 1999;27(3):405-16

41. Cohen S, Kamarck T, Mermelstein R. A global measure of perceived stress. J Health Soc Behav. 1983:24(4):385-96.

42. Edmondson D, Green P, Ye S, Halazun HJ, Davidson KW. Psychological stress and 30-day all-cause hospital readmission in acute coronary syndrome patients: an observational cohort study. PLoS One. 2014;9(3):e91477.

43. Manos RC, Kanter JW, Luo W. The behavioral activation for depression scaleshort form: development and validation. Behav Ther. 2011;42(4):726-39.

44. Zeger SL, Liang KY. Longitudinal data analysis for discrete and continuous outcomes. Biometrics. 1986;42(1):121-30.

45. Benowitz NL, Prochaska JJ. Smoking cessation after acute myocardial infarction. J Am Coll Cardiol. 2013;61(5):533-5.

46. Zuidersma M, Thombs BD, de Jonge P. Onset and recurrence of depression as predictors of cardiovascular prognosis in depressed acute coronary syndrome patients: a systematic review. Psychother Psychosom. 2011;80(4):227-37.

47. Parker GB, Hilton TM, Walsh WF, Owen CA, Heruc GA, Olley A, Brotchie H, Hadzi-Pavlovic D. Timing is everything: the onset of depression and acute coronary syndrome outcome. Biol Psychiatry. 2008;64(8):660-6.

\section{Submit your next manuscript to BioMed Central and we will help you at every step:}

- We accept pre-submission inquiries

- Our selector tool helps you to find the most relevant journal

- We provide round the clock customer support

- Convenient online submission

- Thorough peer review

- Inclusion in PubMed and all major indexing services

- Maximum visibility for your research

Submit your manuscript at www.biomedcentral.com/submit
) Biomed Central 